\title{
Beam Ion Instability in the Ultimate Storage Ring
}

\author{
L. Wang \\ SLAC National Accelerator Laboratory, Menlo Park, CA, USA \\ Abstract
}

Ultimate storage ring light sources have electron emittances near the X-ray diffraction limit. Beam ion instability is a great concern for such ultra-small emittance rings. Beam ion instability in the ultimate storage ring belongs to a special regime where most ions are not trapped along the bunch train. This paper investigates the beam-ion dynamics in such special regimes.

PACS numbers: 29.27.Bd, 29.20.db

Keywords: instability, space charge field, vacuum, ion trapping

\section{INTRODUCTION}

There is worldwide interest in developing so-called Ultimate Storage Ring (USR) light sources having electron emittances near the X-ray diffraction limit. That would provide spectral brightness one or two orders of magnitude higher than existing third-generation sources and very large coherent flux in the multi-keV photon energy range [1]. At the same time, there is growing scientific interest in X-ray Free Electron Laser (FEL) sources that can provide a continuous train of evenly spaced, low peak power, coherent photon pulses at repetition rates above $1 \mathrm{kHz}$.

A storage ring based on a free-electron laser (FEL) is proposed [2]. Table 1 lists the main parameters of the USR and FEL configuration in PEP-X. There is a small emittance of $12 \mathrm{pm}$-rad in both planes for the USR configuration. To reduce IBS (intra-beam scattering) and increase beam lifetime, the beam is nearly round; therefore, ion instabilities in both planes are expected. The FEL beam is flat, as are most third-generation light sources. However, its vertical emittance is only $1.6 \mathrm{pm}$. There is a high bunch charge and a short bunch length in the FEL configuration in order to provide a peak current above $200 \mathrm{~A}$. A low-alpha model is usually used to generate a short bunch, but the bunch charge is orders of magnitude lower, limited by micro-bunch instabilities. Here the bunch length is assumed to be compressed with high RF voltage. The coupled bunch instability driven by high order modes (HOMs) in the RF cavities limits the bunch number and therefore the beam current. However, the beam current can be improved when the RF gradient is further increased.

In this paper we use PEP-X as one example to study beam ion instability in the ultimate storage ring where the beam can be round or flat. The bunch length in general does not affect the dynamics of beam ion instability in typical electron rings. Therefore, the two configurations of PEP-X here represent round and flat beams in terms of beam ion instability. When the beam emittance decreases, the interaction between the electron bunches and ions becomes stronger. Therefore, it is interesting and also important to study beam ion instability in the ultimate storage rings. Since beam ion instability depends on the transverse beam size, it is necessary to study beam ion instability with both round and flat beams.

Table 1: Main parameters of PEP-X as a conventional ring-based synchrotron light source or a driver of a free electron laser in a bypass. 


\begin{tabular}{|l|c|c|c|}
\hline Parameter & Symbol/Unit & PEP-X(USR) & PEP-X(FEL) \\
\hline Beam energy & $E(\mathrm{GeV})$ & 4.5 & 4.5 \\
\hline Circumference & $C(m)$ & 2200 & 2200 \\
\hline Beam current & $I_{b}(m A)$ & 200 & 200 \\
\hline Bunch charge & $q(n C)$ & 0.5 & 0.75 \\
\hline Transverse emittance & $\varepsilon_{x} / \varepsilon_{y}(\mathrm{pm}-\mathrm{rad})$ & $12 / 12$ & $160 / 1.6$ \\
\hline Energy spread & $\sigma_{E}\left(10^{-3}\right)$ & 1.25 & 1.55 \\
\hline Bunch length & $\sigma_{z}(\mathrm{~mm})$ & 3.0 & 0.3 \\
\hline RF voltage & $V_{R F}(\mathrm{MV})$ & 8.3 & 282 \\
\hline RF frequency & $f_{R F}(\mathrm{MHz})$ & 476 & 1428 \\
\hline Harmonic number & $h$ & 3492 & 10476 \\
\hline Radiation damping time & $\tau_{x} / \tau_{y}(m s)$ & 18 & 18 \\
\hline Bunch number & $\mathrm{Nb}$ & 2935 & 1956 \\
\hline
\end{tabular}

\section{SIMULATIONS}

\subsection{Simulation model}

Time-domain simulations have a number of advantages in the study of beam-ion instabilities: the nonlinearity of the interaction force between electron beam and ion-cloud can be included, the effects of beam optics and bunchtrain gaps with arbitrary beam fill patterns are readily visible, and a realistic vacuum model with multi-gas species is straightforward. A particle-in-cell (PIC) code based on a weak-strong model is used here [3]. The ions are distributed along the whole ring according to the vacuum pressure. The optics of a ring with the MAD [4] type of format is read as an input of the code. The electron bunches are distributed along the accelerator according to a realistic filling pattern, which can be arbitrary in the model. Therefore, the bunch train gaps are automatically included. Each electron bunch is represented by a single macro-particle or a few particles (slice model) in the simulation. New ions are generated in each element when electron bunches pass by according to the local vacuum and electron bunch information. When there are multiple gas species in the vacuum, multiple types of ion species are generated. The initial transverse spatial distribution of the ions is a Gaussian distribution with the same rms size as the electron beam present. But these ions are uniformly distributed in that element along the direction of beam motion. Therefore, this model can treat arbitrary vacuums. For instance, the vacuum can have multiple gas species, and the vacuum pressure can be different at different sections of the accelerator. The ions generated by previous bunches will receive a kicker from the subsequent bunches and the self-field of the ions. Meanwhile, the electron bunch also receives a kicker from the ion-cloud in each element.

The gas composition of the vacuum varies in different accelerators. We assume the vacuum consists of $H_{2}(48 \%)$, $\mathrm{CH}_{4}(5 \%), \mathrm{H}_{2} \mathrm{O}(16 \%), \mathrm{CO}(14 \%)$, and $\mathrm{CO}_{2}(17 \%)$. The gas species have a component similar to the vacuum in 
SPEAR3[5]. We further assume a total pressure of 0.4 nTorr everywhere along the ring. We will use this vacuum model throughout this paper in both simulation and analysis.

\subsection{USR beam}

The USR beam has 10 bunch trains with 2935 bunches in total. The total RF buckets is 3492 . The electron bunches are close to, but not exactly, uniformly filled. Five of the bunch trains have 293 bunches, and the remainder have 294 bunches. The bunch spacing along the bunch train is $2.1 n s$ (1 RF bucket spacing). Three of the bunch trains have a gap of $55 \mathrm{RF}$ buckets spacing while the other seven have a gap of $56 \mathrm{RF}$ buckets spacing. Unlike most existing storage light source, the horizontal and vertical emittances of the USR beam are the same; therefore, the beam is nearly round, and both horizontal and vertical beam instabilities are expected.

Fig. 1 shows the horizontal and vertical beam instability driven by ions. The oscillation amplitudes of individual bunches are shown. The amplitude of the beam is always small, which is characteristic of beam ion instability. The instability in the vertical plane is faster than in the horizontal one due to the smaller betatron function in the vertical plane. There is at first an exponential growth in instability, which is eventually slowed down by the nonlinearity of space charge forces when the amplitude is larger than the beam size. This can be more clearly seen in Fig. 2, where the amplitude is plotted in a logarithmic scale. The exponential growth time is $0.64 \mathrm{~ms}$ and $0.26 \mathrm{~ms}$ for the horizontal and vertical directions, respectively.

Fig. 3 shows the unstable modes of the instabilities. Instead of the unstable mode number, their frequencies are plotted for direct comparison with calculated impedance. The unstable modes have frequencies between $0 \sim 100 \mathrm{MHz}$, which are below the frequencies of RF HOMs. The frequencies are higher than the observed ones in existing light sources, for instance SPEAR3 [5], because of the small beam size in USR. The several spikes in the frequency distribution are the effects of different ions in different locations along the ring. This can be clearly explained by the calculated impedance driven by ions as shown later. The larger the variation of the beam size along the ring, the wider the beam spectrum of the unstable modes.
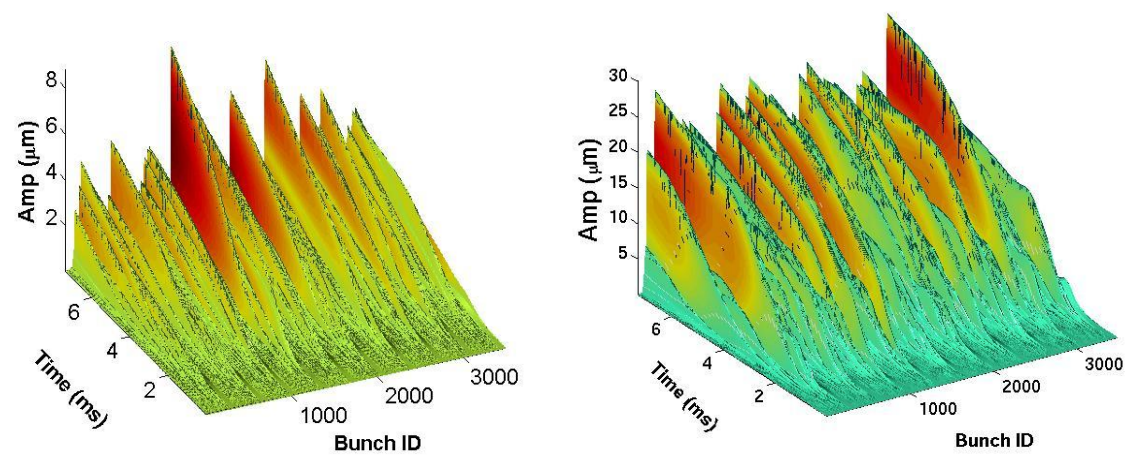

Fig. 1. The horizontal (left) and vertical (right) beam ion instabilities of a USR beam. The bunch number is 2935 with a filling pattern of 10 bunch trains. Zero chromaticity and total vacuum pressure of 0.4 nTorr are used in the simulation. Radiation damping is not included. 


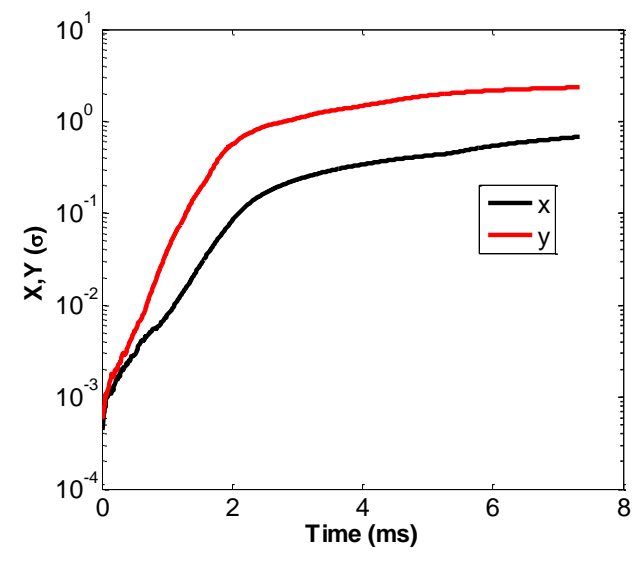

Fig. 2. The beam ion instability of a USR beam. The vertical axis shows the fastest growth of amplitude in the unit of beam size.
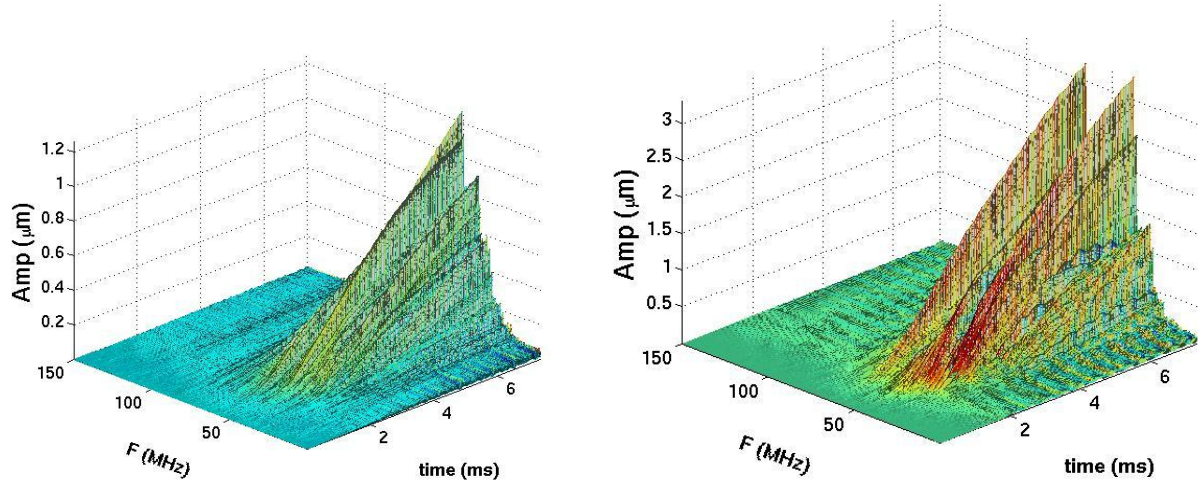

Fig. 3. Unstable modes of the horizontal (left) and vertical (right) beam ion instabilities for a USR beam. Instead of the mode number, the frequency of the modes is plotted for later comparison with calculated impedance.

\subsection{FEL beam}

A high peak current, about 200A, is required for FEL. We assume this can be done by combining high RF voltage with high RF frequency. The required voltage is $282 M V$ with tripled RF frequency. Preliminary study shows that the total beam current is limited to $20 \mathrm{~A}$ due to the HOMs in the cavities. Nevertheless, we assume a beam current of $200 \mathrm{~mA}$ in the estimation of beam ion instability. The FEL beam has 10 bunch trains with a total of 1956 bunches. The filling pattern of electron bunches is close to uniform, with a bunch spacing of $2.1 \mathrm{~ns}$. Four of the bunch trains have 195 bunches, and the remainder have 196 bunches. Two of the bunch train gaps are $322 n s$ while the others are 322.7ns. The FEL beam is flat with a small vertical emittance. The horizontal and vertical emittances are 160 and 1.6 pm-rad, respectively. Figure 4 shows the beam ion instability of the EFL beam. The exponential growth times are $4.9 \mathrm{~ms}$ and $0.16 \mathrm{~ms}$ in the horizontal and vertical planes, respectively. There is weak instability in the horizontal plane due to the larger horizontal emittance. However, the horizontal instability is still faster than the radiation damping time of $18 \mathrm{~ms}$. 

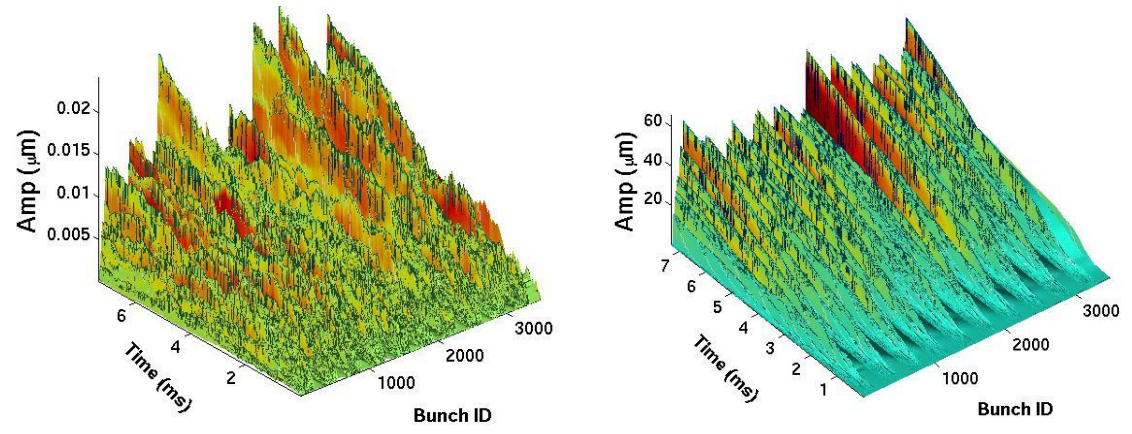

Fig. 4. The horizontal (left) and vertical (right) beam ion instabilities of the FEL beam. The bunch number is 1956 with a 10 bunch trains filling pattern. A total vacuum pressure of 0.4 nTorr is used in the simulation.

\section{ANALYSIS}

When the separation between electron bunch and ions is smaller compared with the beam size, the interaction force between them is linear and can therefore be well represented by a wake field, as [3]

$$
W_{\text {ring }}(s)=\int_{0}^{c} \frac{4}{3} \frac{\omega_{i, y}(z)}{c} \frac{\lambda_{i}(z) S_{b}}{N_{e}} \frac{1}{\sigma_{y}(z)\left(\sigma_{y}(z)+\sigma_{x}(z)\right)} e^{-\frac{\omega_{i}(z) s}{2 Q_{0} c}} \sin \left(\frac{\omega_{i}(z) s}{c}\right) d z,
$$

where $N_{e}$ is the number of electrons per bunch, $S_{b}$ is bunch spacing and $\sigma_{x, y}$ is the transverse $r m s$ beam size of the electron bunches, $c$ is the speed of light, $\lambda_{i}$ is the ion line density, and $\omega_{i}$ is the ion oscillation frequency. $Q_{0}$ is an effective quality factor due to the nonlinear space charge field, which depends on the distribution of the particles. For a Gaussian distributed beam, $Q_{0}$ is about 9 [3]. The integral is done for the whole ring; therefore, the wake fields are the contributions of all ions along the whole ring. In the linear regime, the coherent ion oscillation frequency is

$$
\omega_{i, y}=2 \pi f_{i, y} \approx c\left(\frac{4 N_{e} r_{p}}{3 A S_{b}\left(\sigma_{x}+\sigma_{y}\right) \sigma_{y}}\right)^{1 / 2} .
$$

Here $r_{p}$ is the classical radius of the proton and $A$ is the mass number of the ion. As shown in Eq. (1), the wake field becomes stronger when the beam size is smaller; therefore, a stronger instability may occur.

Fig. 5 is the calculated vertical impedance of the ion cloud with the assumption that the motion of ions along the bunch train is stable while the ion density along the bunch train gap decays exponentially, with a decay time order of the ion's oscillation period. The impedance is obtained by the Fourier transform of the wake field. For each type of ion, there are two separated regimes in their frequencies, which are the contributions of ions in the arc and the straight section, respectively. The large difference of the betatron functions in the two sections results in the ion frequencies being well separated. Furthermore, the impedance due to $\mathrm{CO}_{2}$ ion has the largest peak at $40 \mathrm{MHz}$. The heavier ions are important for beam ion instability due to their large ionization cross-section, slower kinetic velocity, and consequently more stable motion in the periodic trapping potential [3]. The highest frequency is above $600 \mathrm{MHz}$, which is the contribution of the $\mathrm{H}^{+}$ions in the low betatron function sections. The impedance shown in Fig. 5 gives a clear picture of the effect of different ion species (vacuum) and beam optics. The large variation in beam size along 
the ring and the multiple gas species in the vacuum make broadband impedance. A broad beam spectrum is observed in SPEAR3 [5].

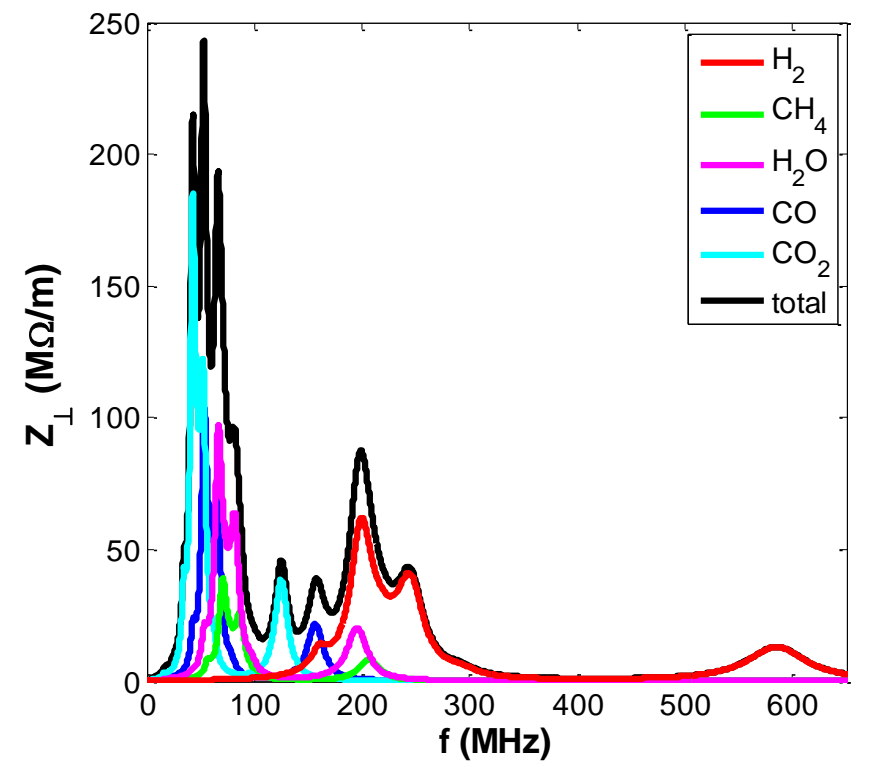

Fig. 5. Real part of vertical impedance driven by different ion species for USR beam. The beam and vacuum are the same as used in the simulation shown in Fig. 1.

When the beam is evenly filled around the ring, the exponential growth rate of the coupled bunch instability for mode $y_{j}^{\mu} \propto e^{2 \pi u j / n_{b}}$ is given by the imaginary component of the coherent frequency shift with mode number $\mu$ [6]

$$
\Omega_{\mu}-\omega_{\beta}=i \frac{N_{e} M r_{e} c}{2 \gamma T_{0}^{2} \omega_{\beta}} \sum_{p=-\infty}^{\infty} Z_{\perp}\left(\left(p M+v_{y}+\mu\right) \omega_{0}+m \omega_{s}\right)
$$

Here $T_{0}$ and $\omega_{0}$ are the revolution period and frequency, $v_{y}$ is the betatron tune, $M$ is the bunch number, and $\omega_{\beta}$ and $\omega_{s}$ are the betatron and synchrotron frequencies. The real part of the frequency shift gives a tune shift, and the imaginary part gives the exponential growth rate of the coupled bunch beam-ion instability. Growth time is the inverse of growth rate. Fig. 6 shows the estimated instability growth rate based on the impedance shown in Fig. 5. The electron beam is approximated with a uniform filling pattern. The fastest unstable mode has a growth time of $0.254 \mathrm{~ms}$, which agrees very well with the simulated growth time of $0.26 \mathrm{~ms}$. It can be clearly seen from Fig. 6 that the most unstable mode is driven by the $\mathrm{CO}$ and $\mathrm{CO}_{2}$ ions. The spikes in the simulated unstable modes (Fig. 3) can be explained by the impedance: the unstable modes are mainly driven by $\mathrm{CO}$ and $\mathrm{CO}_{2}$ ions in the large beam size sections. The calculations for the FEL beam also agree with the simulation. 


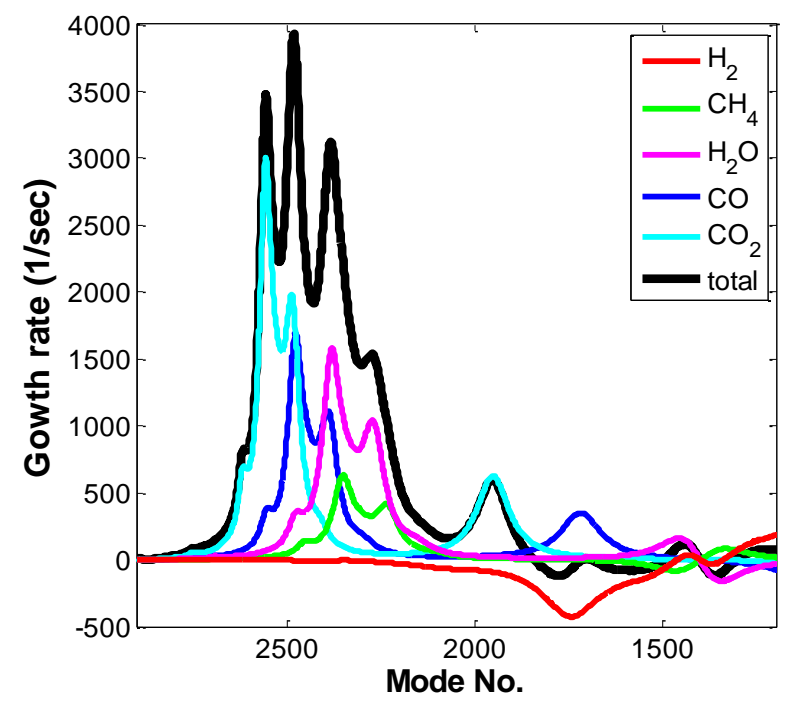

FIG. 6. Estimated vertical instability growth rate of USR beam from the ion impedance shown in Fig. 5.

However, the simulated beam spectrum (Fig.3) does not completely agree with theory. For instance, the unstable modes driven by impedance with a frequency higher than $100 \mathrm{MHz}$ are not seen in the simulation. The existing theories assume that the motion of ion along the bunch train is stable, $\omega_{i} S_{b} / c<<1$. In this case, the beam can be treated as a coasting beam. However, the coasting model fails when the bunch spacing is too long compared with the ion oscillation period. To be more specific, if the electron bunches are uniformly filled, the stable condition of ion motion in the linear theory is

$$
S_{b}<\frac{2\left(\sigma_{x}+\sigma_{y}\right) \sigma_{y} A}{N_{e} r_{p}}=\sqrt{\frac{8}{3}} \frac{c}{\omega_{i}}
$$

In other words, it requires $\omega_{i} S_{b} / c<1.6$. This gives a threshold of ion frequency for stable motion along the bunch train as $121 \mathrm{MHz}$ for PEP-X. Ions with frequencies higher than this threshold will be over-forced and escape from the electron bunch gap. The electron bunches work like focusing elements, similar to the quadruple magnet. When the focusing is too strong, ion motion becomes unstable. The ultra-strong bunched beam works just like a clearing electrode in this regime. Under this extreme condition, the existing theories do not apply. The ultra-low emittance beam destabilizes ion motion, thereby making the instability relatively weaker, which is one special advantage of ultra-low emittance beams. However, when the beam emittance increases, more ions can be trapped and the beam instability may become even stronger. Beam ion instability should be carefully investigated whenever the beam conditions change, especially beam emittance, bunch spacing, and beam current. For the current PEP-X parameters, ions along part of the ring (the small betatron function section) are un-trapped by the bunch train. For instance, the impedance shown in Fig. 5 with frequency above $121 \mathrm{MHz}$ is overestimated when the stable condition of the ion is considered. Even the heavy $\mathrm{CO}_{2}$ ions at smaller betatron sections are not deeply trapped. However, the impedance at lower frequencies is still accurate; and it drives the most unstable modes. This explains why the calculated fastest growth time still agrees with simulations. 


\section{SUMMARY AND DISCUSSION}

This paper summarizes the comprehensive analyses and simulations of the beam-ion instabilities in an ultimate storage ring with a round and flat beam. There are both strong horizontal and vertical beam instabilities for a round beam, and the horizontal instability becomes weaker when the beam is flat. The beam ion instability in the ultimate storage rings is at the same level of existing light sources, about $0.1 \sim 0.2 \mathrm{~ms}$ for a total vacuum pressure of $0.4 n$ Torr. The multiple bunch train filling pattern in an ultra-low emittance ring becomes much more effective in mitigating beam ion instability by reducing the ion trapping along the train gap. In other words, the instability growth rate is roughly proportional to the bunch train length [3]. Multiple bunch train beam with short bunch train gap is a very

effective mitigation for such ultra-low emittance beam. Furthermore, the ions from the low betatron function sections of the ring are not trapped, even along the bunch train. This mechanism also weakens ion instability, although it does not affect the fastest growth mode, which is driven by $\mathrm{CO}$ and $\mathrm{CO}_{2}$ ions in the large beam size section. When the beam intensity further increases, the ions in the whole ring may become unstable. Then the beam ion instability belongs to a special regime: instability with un-trapped ions where the existing linear theories do not apply.

\section{ACKNOWLEDGEMENTS}

This work is supported by the US Department of Energy Contract No. DE-AC02-76SF00515.

\section{REFERENCES}

[1] R. Hettel and Q. Qin, "Report on the Workshop on Accelerator R\&D for Ultimate Storage Rings", Oct. 30 - Nov. 1, 2012, Beijing, China, SLAC-PUB-15379, February (2013).

[2] Yunhai Cai, Yuantao Ding, Robert Hettel, Zhirong Huang, Lanfa Wang, Liling Xiao, “An X-ray Free Electron Laser Driven by an Ultimate Storage Ring”, Synchrotron Radiation News, Vol.26, No.3,p39, 2013

[3] L. Wang, Y. Cai, T. O. Raubenheimer and H. Fukuma, Phys. Rev. ST Accel. Beams 14, 084401 (2011)

[4] D. J. Peake, et.al., in Proceedings of 2009 Particle Accelerator Conference, Vancouver, Canada, 4105(2009)

[5] L. Wang, J. Safranek, Y. Cai, J. Corbett, R. O. Hettel, T. O. Raubenheimer, J. Schmerge and J. Sebek, Phys. Rev. ST Accel. Beams , 16, 104402 (2013)

[6] A. Chao, "Physics of Collective Beam Instabilities in High Energy Accelerators", Wiley, (1995). 